\title{
Complete angular distribution measurements of two-body deuteron photodisintegration between 0.5 and $3 \mathrm{GeV}$
}

M. Mirazita, ${ }^{1}$ F. Ronchetti, ${ }^{1,2}$ P. Rossi, ${ }^{1}$ E. De Sanctis, ${ }^{1}$ G. Adams, ${ }^{29}$ P. Ambrozewicz, ${ }^{12}$ E. Anciant, ${ }^{4}$ M. Anghinolfi, ${ }^{16}$ B. Asavapibhop, ${ }^{22}$ G. Audit, ${ }^{4}$ H. Avakian, ${ }^{1,34}$ H. Bagdasaryan, ${ }^{27}$ J. P. Ball, ${ }^{3}$ S. Barrow, ${ }^{13}$ M. Battaglieri, ${ }^{16}$ K. Beard, ${ }^{19}$ M. Bektasoglu, ${ }^{27}$ M. Bellis, ${ }^{29}$ N. Benmouna, ${ }^{14}$ B. L. Berman, ${ }^{14}$ W. Bertozzi, ${ }^{21}$ N. Bianchi, ${ }^{1}$ A. S. Biselli, ${ }^{29}$

S. Boiarinov, ${ }^{34,18}$ B. E. Bonner, ${ }^{30}$ S. Bouchigny, ${ }^{17,34}$ R. Bradford, ${ }^{6}$ D. Branford, ${ }^{11}$ W. J. Briscoe, ${ }^{14}$ W. K. Brooks, ${ }^{34}$ V. D. Burkert, ${ }^{34}$ C. Butuceanu, ${ }^{38}$ J. R. Calarco, ${ }^{24}$ D. S. Carman, ${ }^{26}$ B. Carnahan, ${ }^{7}$ S. Chen, ${ }^{13}$ P. L. Cole, ${ }^{33,34}$ D. Cords, ${ }^{34}$ P. Corvisiero, ${ }^{16}$ D. Crabb, ${ }^{37}$ H. Crannell, ${ }^{7}$ J. P. Cummings, ${ }^{29}$ R. De Vita, ${ }^{16}$ P. V. Degtyarenko, ${ }^{34}$ H. Denizli, ${ }^{28}$ L. Dennis, ${ }^{13}$ A. Deppman, ${ }^{1}$ K. V. Dharmawardane, ${ }^{27}$ K. S. Dhuga, ${ }^{14}$ C. Djalali, ${ }^{32}$ G. E. Dodge, ${ }^{27}$ D. Doughty, ${ }^{8,34}$ P. Dragovitsch, ${ }_{13}^{13}$ M. Dugger, ${ }^{3}$ S. Dytman, ${ }^{28}$ O. P. Dzyubak, ${ }^{32}$ H. Egiyan, ${ }^{38,34}$ K. S. Egiyan, ${ }^{39}$ L. Elouadrhiri, ${ }^{34}$ A. Empl, ${ }^{29}$

P. Eugenio, ${ }^{13}$ R. Fatemi, ${ }^{37}$ R. J. Feuerbach, ${ }^{6}$ J. Ficenec, ${ }^{36}$ T. A. Forest, ${ }^{27}$ H. Funsten, ${ }^{38}$ M. Gai, ${ }^{9}$ G. Gavalian, ${ }^{24,39}$

S. Gilad, ${ }^{21}$ G. P. Gilfoyle, ${ }^{31}$ K. L. Giovanetti, ${ }^{19}$ C. I. O. Gordon, ${ }^{15}$ K. Griffioen, ${ }^{38}$ M. Guidal, ${ }^{17}$ M. Guillo, ${ }^{32}$ L. Guo, ${ }^{34}$ V. Gyurjyan, ${ }^{34}$ C. Hadjidakis, ${ }^{17}$ R. S. Hakobyan, ${ }^{7}$ J. Hardie, ${ }^{8,34}$ D. Heddle, ${ }^{8,34}$ F. W. Hersman, ${ }^{24}$ K. Hicks, ${ }^{26}$ R. S. Hicks, ${ }^{22}$ M. Holtrop, ${ }^{24}$ J. Hu, ${ }^{29}$ C. E. Hyde-Wright,${ }^{27}$ Y. Ilieva, ${ }^{14}$ M. M. Ito, ${ }^{34}$ D. Jenkins, ${ }^{36}$ K. Joo, ${ }^{34,37}$ J. D. Kellie, ${ }^{15}$

M. Khandaker, ${ }^{25}$ K. Y. Kim, ${ }^{28}$ K. Kim, ${ }^{20}$ W. Kim, ${ }^{20}$ A. Klein, ${ }^{27}$ F. J. Klein, ${ }^{7,34}$ A. V. Klimenko, ${ }^{27}$ M. Klusman, ${ }^{29}$ M. Kossov, ${ }^{18}$ L. H. Kramer, ${ }^{12,34}$ J. Kuhn, ${ }^{6}$ S. E. Kuhn, ${ }^{27}$ J. Kuhn, ${ }^{6}$ J. Lachniet, ${ }^{6}$ J. M. Laget, ${ }^{4}$ D. Lawrence, ${ }^{22}$ Ji Li, ${ }^{29}$ A. C. S. Lima, ${ }^{14}$ K. Livingston, ${ }^{15}$ K. Lukashin, ${ }^{34}$ J. J. Manak, ${ }^{34}$ C. Marchand, ${ }^{4}$ S. McAleer, ${ }^{13}$ J. McCarthy, ${ }^{37}$ J. W.C. McNabb, ${ }^{6}$

B. A. Mecking, ${ }^{34}$ S. Mehrabyan, ${ }^{28}$ J. J. Melone, ${ }^{15}$ M. D. Mestayer, ${ }^{34}$ C. A. Meyer, ${ }^{6}$ K. Mikhailov, ${ }^{18}$ R. Miskimen, ${ }^{22}$ V. Mokeev, ${ }^{23,34}$ L. Morand, ${ }^{4}$ S. A. Morrow, ${ }^{17}$ V. Muccifora, ${ }^{1}$ J. Mueller, ${ }^{28}$ G. S. Mutchler, ${ }^{30}$ J. Napolitano, $^{29}$ R. Nasseripour, ${ }^{12}$ S. Niccolai, ${ }^{14}$ G. Niculescu, ${ }^{26}$ I. Niculescu, ${ }^{14}$ B. B. Niczyporuk, ${ }^{34}$ R. A. Niyazov, ${ }^{34}$ M. Nozar, ${ }^{34}$ J. T. O'Brien, ${ }^{7}$

G. V. O'Rielly, ${ }^{14}$ M. Osipenko, ${ }^{23}$ A. Ostrovidov, ${ }^{13}$ K. Park, ${ }^{20}$ E. Pasyuk, ${ }^{3}$ G. Peterson, ${ }^{22}$ S. A. Philips, ${ }^{14}$ N. Pivnyuk, ${ }^{18}$ D. Pocanic, ${ }^{37}$ O. Pogorelko, ${ }^{18}$ E. Polli, ${ }^{1}$ S. Pozdniakov, ${ }^{18}$ B. M. Preedom, ${ }^{32}$ J. W. Price, ${ }^{5}$ Y. Prok, ${ }^{37}$ D. Protopopescu, ${ }^{24}$ L. M. Qin, ${ }^{27}$ B. A. Raue, ${ }^{12,34}$ G. Riccardi, ${ }^{13}$ G. Ricco, ${ }^{16}$ M. Ripani, ${ }^{16}$ B. G. Ritchie, ${ }^{3}$ G. Rosner, ${ }^{15}$ D. Rowntree, ${ }^{21}$

P. D. Rubin, ${ }^{31}$ F. Sabatié, ${ }^{4,27}$ C. Salgado, ${ }^{25}$ J. P. Santoro, ${ }^{36,34}$ V. Sapunenko, ${ }^{16}$ R. A. Schumacher, ${ }^{6}$ V. S. Serov, ${ }^{18}$ Y. G. Sharabian, ${ }^{34,39}$ J. Shaw, ${ }^{22}$ S. Simionatto, ${ }^{14}$ A. V. Skabelin, ${ }^{21}$ E. S. Smith, ${ }^{34}$ L. C. Smith, ${ }^{37}$ D. I. Sober, ${ }^{7}$ M. Spraker, ${ }^{10}$ A. Stavinsky, ${ }^{18}$ S. Stepanyan, ${ }^{27,39}$ B. Stokes, ${ }^{13}$ P. Stoler, ${ }^{29}$ I. I. Strakovsky, ${ }^{14}$ S. Strauch, ${ }^{14}$ M. Taiuti, ${ }^{16}$ S. Taylor, ${ }^{30}$

D. J. Tedeschi, ${ }^{32}$ U. Thoma, ${ }^{34}$ R. Thompson, ${ }^{28}$ A. Tkabladze, ${ }^{26}$ L. Todor, ${ }^{6}$ C. Tur, ${ }^{32}$ M. Ungaro, ${ }^{29}$ M. F. Vineyard, ${ }^{35}$ A. V. Vlassov, ${ }^{18}$ K. Wang, ${ }^{37}$ L. B. Weinstein, ${ }^{27}$ H. Weller, ${ }^{10}$ D. P. Weygand, ${ }^{34}$ C. S. Whisnant, ${ }^{32}$ E. Wolin, ${ }^{34}$ M. H. Wood, ${ }^{32}$ A. Yegneswaran, ${ }^{34}$ J. Yun, ${ }^{27}$ B. Zhang, ${ }^{21}$ and Z. Zhou ${ }^{21}$

(CLAS Collaboration)

${ }^{1}$ Istituto Nazionale di Fisica Nucleare, Laboratori Nazionali di Frascati, PO 13, 00044 Frascati, Italy

${ }^{2}$ Università di ROMA III, 00146 Roma, Italy

${ }^{3}$ Arizona State University, Tempe, Arizona 85287, USA

${ }^{4}$ CEA-Saclay, Service de Physique Nucléaire, F91191 Gif-sur-Yvette, Cedex, France

${ }^{5}$ University of California at Los Angeles, Los Angeles, California 90095, USA

${ }^{6}$ Carnegie Mellon University, Pittsburgh, Pennsylvania 15213, USA

${ }^{7}$ Catholic University of America, Washington, D.C. 20064, USA

${ }^{8}$ Christopher Newport University, Newport News, Virginia 23606, USA

${ }^{9}$ University of Connecticut, Storrs, Connecticut 06269, USA

${ }^{10}$ Duke University, Durham, North Carolina 27708, USA

${ }^{11}$ Edinburgh University, Edinburgh EH9 3JZ, United Kingdom

${ }^{12}$ Florida International University, Miami, Florida 33199, USA

${ }^{13}$ Florida State University, Tallahassee, Florida 32306, USA

${ }^{14}$ The George Washington University, Washington, D.C. 20052, USA

${ }^{15}$ University of Glasgow, Glasgow G12 8QQ, United Kingdom

${ }^{16}$ Istituto Nazionale di Fisica Nucleare, Sezione di Genova, 16146 Genova, Italy

${ }^{17}$ Institut de Physique Nucleaire ORSAY, IN2P3 B.P. 1, 91406 Orsay, France

${ }^{18}$ Institute of Theoretical and Experimental Physics, Moscow, 117259, Russia

${ }^{19}$ James Madison University, Harrisonburg, Virginia 22807, USA

${ }^{20}$ Kyungpook National University, Taegu 702-701, South Korea

${ }^{21}$ Massachusetts Institute of Technology, Cambridge, Massachusetts 02139, USA

${ }^{22}$ University of Massachusetts, Amherst, Massachusetts 01003, USA

${ }^{23}$ Moscow State University, 119899 Moscow, Russia

${ }^{24}$ University of New Hampshire, Durham, New Hampshire 03824, USA

${ }^{25}$ Norfolk State University, Norfolk, Virginia 23504, USA

${ }^{26}$ Ohio University, Athens, Ohio 45701, USA

${ }^{27}$ Old Dominion University, Norfolk, Virginia 23529, USA

${ }^{28}$ University of Pittsburgh, Pittsburgh, Pennsylvania 15260, USA 


\author{
${ }^{29}$ Rensselaer Polytechnic Institute, Troy, New York 12180, USA \\ ${ }^{30}$ Rice University, Houston, Texas 77005, USA \\ ${ }^{31}$ University of Richmond, Richmond, Virginia 23173, USA \\ ${ }^{32}$ University of South Carolina, Columbia, South Carolina 29208, USA \\ ${ }^{33}$ University of Texas at El Paso, El Paso, Texas 79968, USA \\ ${ }^{34}$ Thomas Jefferson National Accelerator Facility, Newport News, Virginia 23606, USA \\ ${ }^{35}$ Union College, Schenectady, New York 12308, USA \\ ${ }^{36}$ Virginia Polytechnic Institute and State University, Blacksburg, Virginia 24061, USA \\ ${ }^{37}$ University of Virginia, Charlottesville, Virginia 22901, USA \\ ${ }^{38}$ College of William and Mary, Williamsburg, Virginia 23187, USA \\ ${ }^{39}$ Yerevan Physics Institute, 375036 Yerevan, Armenia
}

(Received 12 May 2004; published 16 July 2004)

\begin{abstract}
Nearly complete angular distributions of the two-body deuteron photodisintegration differential cross section have been measured using the CEBAF Large Acceptance Spectrometer detector and the tagged photon beam at the Thomas Jefferson National Accelerator Facility. The data cover photon energies between 0.5 and $3.0 \mathrm{GeV}$ and center-of-mass proton scattering angles $10^{\circ}-160^{\circ}$. The data show a persistent forward-backward angle asymmetry over the explored energy range, and are well described by the nonperturbative quark gluon string model.
\end{abstract}

DOI: 10.1103/PhysRevC.70.014005

PACS number(s): 24.85.+p, 25.20.-x, 21.45.+v

\section{INTRODUCTION}

Quantum chromodynamics (QCD) has been successfully applied in describing the structure and production of hadrons at high energies where perturbation theory can be used. There one can derive QCD scaling laws for the cross sections and hadronic helicity conservation laws. However, nuclear reactions have been conventionally described in terms of baryons and mesons rather than quarks and gluons. It is therefore interesting and important to know in which energy region the transition from hadronic picture to quark-gluon picture takes place. This is why major efforts in nuclear physics have been devoted, both theoretically and experimentally, to looking for qualitatively new phenomena that arise from the underlying quark degrees of freedom, and that cannot be modeled using meson field theories.

Deuteron photodisintegration at high energies is especially suited for this study, because a relatively large amount of momentum is transferred to the nucleons for a relatively low incident photon energy [1,2]. One possible signature for the transition from nucleon-meson to quark-gluon degrees of freedom is the scaling of reaction cross sections above some incident photon energy. In particular, simple constituent counting rules (CCR) $[3,4]$ predict an asymptotic $s^{-11}$ dependence of $d \sigma / d t$ of the process at all proton angles. Here $s$ and $t$ are the invariant Mandelstam variables for the total energy squared and the four-momentum transfer squared, respectively.

Deuteron photodisintegration cross sections above $1.2 \mathrm{GeV}$ are available for photon energies $E_{\gamma}$ up to $5 \mathrm{GeV}$ at three center-of-mass proton angles, $\vartheta_{p}^{\text {c.m. }}=36^{\circ}, 52^{\circ}, 69^{\circ}$; up to $4 \mathrm{GeV}$ at $\vartheta_{p}^{\text {c.m. }}=90^{\circ}$ [5-9]; and at eight angles with $\vartheta_{p}^{\text {c.m. }}=30^{\circ}-143^{\circ}$, for $E_{\gamma}=1.6,1.9$, and $2.4 \mathrm{GeV}$ [10]. The asymptotic scaling predicted by CCR is observed at $\vartheta_{p}^{\text {c.m. }}$ $=69^{\circ}$ and $90^{\circ}$ already at $E_{\gamma}=1 \mathrm{GeV}$ and at $\vartheta_{p}^{\text {c.m. }}=52^{\circ}$ and $36^{\circ}$ only from 3 and $4 \mathrm{GeV}$, respectively. In contrast, polarization observables measured at $\vartheta_{p}^{\text {c.m. }}=90^{\circ}$ for photon ener- gies up to $2 \mathrm{GeV}[11,12]$ do not support hadronic helicity conservation predicted by perturbative QCD (pQCD). Thus, it seems that although the observation of the scaling in the cross section at a few proton angles indicates the onset of the quark-gluon degrees of freedom, the appropriate underlying physics has a mixture of perturbative and nonperturbative QCD aspects.

In this context, several non-pQCD models attempt to account for the experimental results using different strategies. The reduced nuclear amplitude model (RNA) [13] incorporates some of the soft physics not described by pQCD by using experimentally determined nucleon form factors to describe the gluon exchanges within the nucleons. The RNA calculation is only available at $\vartheta_{p}^{\text {c.m. }}=90^{\circ}$ and makes no predictions for the angular dependence of the cross section. The calculations are normalized to data at energies sufficiently large, assuming that perturbative regime is reached.

The hard quark rescattering mechanism model (HRM) $[14,15]$ assumes that the photon is absorbed by a quark in one nucleon, followed by a high momentum transfer with a quark of the other nucleon leading to the production of two nucleons with high relative momentum. The nuclear scattering amplitude is expressed as a convolution of the large $p n$ scattering amplitude, the hard photon-quark interaction vertex, and the low-momentum nuclear wave function. The authors use experimental data for the $p n$ cross section, but since data do not exist for the actual kinematic conditions needed, they must be extrapolated, and predictions for deuteron photodisintegration are given as a band corresponding to the uncertainties introduced by the extrapolations. The model provides a parameter-free prediction of $d \sigma / d t$ at $\vartheta_{p}^{\text {c.m. }}=90^{\circ}$, and introduces a phenomenological function $f(t / s)$ that is close to unity at $\vartheta_{p}^{\text {c.m. }}=90^{\circ}$, and varies slowly with $\vartheta_{p}^{\text {c.m. }}$. Another attempt [16] to describe the deuteron photodisintegration within the same theoretical framework of HRM, using an exact calculation of the quark exchange am- 
plitude, provides evidence that the assumption used in Refs. $[14,15]$ are questionable.

The quark-gluon string model (QGS) [17-19] describes the reaction as proceeding through three-quark exchange, with an arbitrary number of gluon exchanges. The exchanged nucleon is replaced by a nucleon Regge trajectory that represents the sum of a tower of exchanged nucleon resonances. The best description of the data is obtained using a nonlinear Regge trajectory. The model takes all but two of its free parameters from other processes, and fixes the remaining two using the experimental data on the deuteron photodisintegration cross section at $E_{\gamma}=1.6 \mathrm{GeV}$ and $\vartheta_{p}^{\text {c.m. }}=36^{\circ}$ and $52^{\circ}$. It provides the angular distributions and polarization observables for few-GeV beam energies.

Despite appearances, hard deuteron photodisintegration is an intractable problem in meson-baryon theories. The asymptotic meson exchange current model (AMEC) [20] is able to extrapolate the conventional $N$ - $\pi$ interaction mechanisms to higher energy using form factors to describe the $d N N$ interaction vertex, and fix an overall normalization factor by fitting the experimental data at $1 \mathrm{GeV}$.

A better insight into the competing models can be obtained from more detailed angular distributions of differential cross sections over broader angular and energy ranges than those presently available, and for final states involving different polarizations of the final hadrons.

We report here the first measurement of nearly complete angular distributions $\left(10^{\circ} \leqslant \vartheta_{p}^{\text {c.m. }} \leqslant 160^{\circ}\right)$ of the two-body deuteron photodisintegration cross section obtained with the CEBAF Large Acceptance Spectrometer (CLAS) in Hall B at the Thomas Jefferson National Accelerator Facility (experiment E93-017) [21] for photon energies between 0.5 and $3 \mathrm{GeV}$. The data offer the opportunity for a detailed study of the energy dependence of the differential cross section of the reaction at fixed proton angles, aiming at determining the onset of asymptotic scaling [22].

In the following, we first give some details of the experiment (Sec. II) and its data analysis (Sec. III). Then, we present our results on the deuteron photodisintegration cross sections $d \sigma / d \Omega$ and $d \sigma / d t$, and compare them to available theoretical models and existing data (Sec. IV). We conclude with a summary (Sec. V).

\section{EXPERIMENTAL SETUP}

The data described in this paper were collected at the Thomas Jefferson National Accelerator Facility (JLab) during a 32-day run in August and September 1999 using the Hall B tagged photon beam [23] and the CLAS detector [24]. The bremsstrahlung photon beam was produced by a 10-13 nA continuous electron beam of energy $E_{0}$ $=2.5 \mathrm{GeV}$ (August) and $3.1 \mathrm{GeV}$ (September) impinging on a gold foil of $10^{-4}$ radiation lengths. A tagging spectrometer, with an energy resolution of $0.1 E_{0} \%$, was used to tag $\sim 10^{7}$ photons per second in the energy range $(0.20-0.95) E_{0}$.

A cylindrical Mylar cryogenic target $10 \mathrm{~cm}$ long and $4 \mathrm{~cm}$ in diameter was filled with liquid deuterium at about $23.7 \mathrm{~K}$. The final-state particles were detected in the CLAS spectrometer, which is built around six superconducting coils producing a toroidal magnetic field symmetric about the beam and oriented primarily in the azimuthal direction. The coils naturally separate the detector into six sectors, each functioning as an independent magnetic spectrometer. Each sector is instrumented with three sets of multiwire drift chambers for track reconstruction and one layer of scintillator counters, covering the angular range from $8^{\circ}$ to $143^{\circ}$, for time-of-flight measurements. The forward region $\left(8^{\circ} \leqslant \vartheta\right.$ $\leqslant 45^{\circ}$ ) contains gas-filled threshold Cherenkov counters and lead-scintillator sandwich-type electromagnetic calorimeters for particle identification. For two CLAS sectors the coverage of the electromagnetic calorimeters is extended up to polar angles of $70^{\circ}$. The trigger for the data acquisition was defined by the coincidence between a signal in the tagger (identified photon) and one charged hadron in CLAS. Under these conditions $1771 \times 10^{6}$ events were collected.

\section{DATA ANALYSIS}

\section{A. Data selection}

A data quality check was performed to select runs with stable beam and detector performance. First, several runbased parameters normalized to the incident photon flux were required to be constant at the few percent level from run to run: (a) the total number of charged particles, and (b) the number of particles $p, \pi^{+}, \pi^{-}, K^{+}$, and $K^{-}$. Then, (c) the number of triggers with at least one charged particle in the final state for each tagger-timing counter, (d) the number of photodisintegration events per $100 \mathrm{MeV}$, and (e) the number of photodisintegration events per CLAS sector were required to be stable within the statistical errors.

After applying the above data quality criteria, about $7 \%$ of the originally collected data had been discarded.

\section{B. Event selection}

Photodisintegration events $\gamma d \rightarrow p n$ were identified as follows:

(1) The software coincidence time window between the tagger and CLAS was set to $\pm 1 \mathrm{~ns}$, since the machine electron bunches are separated by $2.004 \mathrm{~ns}$.

(2) Only events with a single charged particle, the proton, in the final state were selected. Protons were identified by determining momentum and path length using the drift chambers, and velocity from the time-of-flight counters.

(3) The reconstructed vertex position of the proton along with beam line was used to remove events originating outside the target cell.

(4) Cuts on the square of the missing mass $M_{X}^{2}=\left(P_{\gamma}\right.$ $\left.+P_{d}-P_{p}\right)^{2}$ were performed to select exclusive two-body deuteron photodisintegration events. Here $P_{\gamma}, P_{d}$, and $P_{p}$ are the four-momenta of the photon, deuteron, and proton, respectively. In this study $M_{X}$ is the mass of the neutron.

\section{Momentum correction}

The momentum of the charged particles measured with CLAS strongly relies on the correct knowledge of the magnetic field geometry and the positioning of the drift cham- 


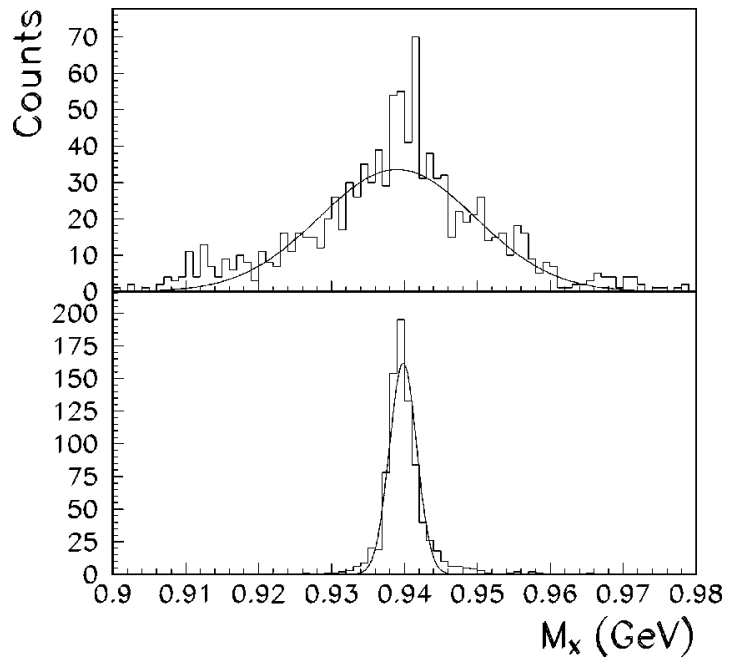

FIG. 1. Distributions for the $M_{X}$ peak values for $\gamma d \rightarrow p X$ events before (top) and after (bottom) the momentum corrections are applied. The width of the corrected distribution is smaller $(13.2 \mathrm{MeV}$ $\mathrm{rms} \rightarrow 3.2 \mathrm{MeV}$ rms) and the centroid is closer to the neutron rest mass.

bers. Due to the complexity of the detector, and particularly of the superconducting torus magnet assembly, it is crucial to make sure that the momentum determined by the drift chamber tracking system is reliable. For this reason the position of the peak of the missing mass distributions from $\gamma d \rightarrow p X$ events has been checked over the whole range of proton momenta and scattering angles. After correcting for the energy loss in the target, the value of the peak was slightly off with respect to the neutron rest mass depending on the proton scattering angle.

A correction procedure has been applied to the data using an empirical function depending only on the measured threemomentum of the proton. It was assumed that the proton track angles are correctly measured, since the CLAS angular resolution is much better than the momentum resolution [24]. We have also checked that the contribution due to the photon energy uncertainty is negligible, by using exclusive $\gamma d$ $\rightarrow p p \pi^{-}$events. The correction function has been calculated for each kinematic bin by fitting the ratio of the expected momentum, as calculated from the photon energy and the proton scattering angle, to the measured momentum [25].

The correction procedure introduced a significant improvement in the resulting width and position of the peak in the missing mass distributions. Figure 1 shows the distributions for the peak values of the $M_{X}$ distributions for $\gamma d$ $\rightarrow p X$ events for all CLAS sectors and all runs, before and after the correction was applied. Clearly, after the correction the distribution of the peak values is sharper (13.2 MeV rms $\rightarrow 3.2 \mathrm{MeV} \mathrm{rms}$ ), and its mean value is closer to the neutron rest mass.

\section{Efficiency}

The single-proton detection efficiency in CLAS cannot be extracted from the deuteron photodisintegration data itself over the whole kinematic region of emitted protons. Exclu-

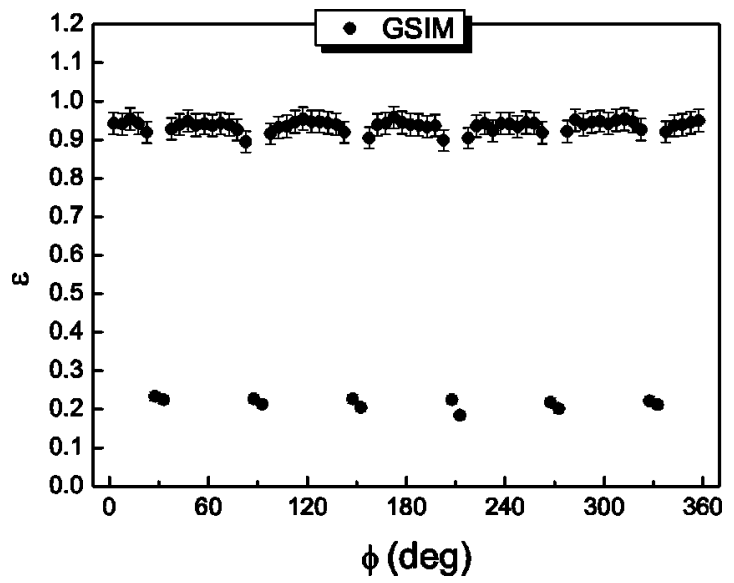

FIG. 2. The CLAS proton detection efficiency evaluated using GSIM as a function of the azimuthal angle $\varphi$ for $P_{p}^{\text {lab }}=0.95 \mathrm{GeV}$, $\vartheta_{p}^{\mathrm{lab}}=65^{\circ}$, and $\varphi$ bins of $5^{\circ}$.

sive events, where both the neutron and the proton are detected, are limited because neutrons could be detected in the calorimeters only over a small angular range $\vartheta^{\mathrm{LAB}} \leqslant 45^{\circ}$ for four CLAS sectors and $\vartheta^{\mathrm{LAB}} \leqslant 70^{\circ}$ for the other two sectors. Other reaction channels with additional particles are problematic because the photodisintegration protons have the highest momentum for a given proton angle and photon energy.

For these reasons, the single proton efficiency has been evaluated using a GEANT simulation (GSIM) of the CLAS detector. Photodisintegration events have been generated uniformly in proton momentum and angle in the laboratory system, and then have been analyzed following the standard reconstruction chain [25].

The proton detection efficiency $\epsilon_{\mathrm{GSIM}}$ has been calculated in each kinematic bin in the laboratory system as the ratio of reconstructed protons $N_{\mathrm{REC}}$ to generated ones $N_{\mathrm{GEN}}$ :

$$
\epsilon_{\mathrm{GSIM}}=\frac{N_{\mathrm{REC}}}{N_{\mathrm{GEN}}} .
$$

Bin widths for proton momentum $\Delta P_{p}^{\mathrm{lab}}=0.1 \mathrm{GeV} / c$ and polar scattering angle $\Delta \vartheta_{p}^{\mathrm{lab}}=10^{\circ}$ have been chosen. A smaller azimuthal angle bin of width $\Delta \varphi_{p}^{\mathrm{lab}}=5^{\circ}$ has been selected to better investigate the azimuthal behavior of the CLAS proton detection efficiency, which gets worse on the boundaries of each sector due to the presence of the magnet coils.

As an example, Fig. 2 shows the resulting proton detection efficiency for $P_{p}^{\mathrm{lab}}=0.95 \mathrm{GeV}$ and $\vartheta_{p}^{\mathrm{lab}}=65^{\circ}$ as a function of the azimuthal angle $\varphi_{p}^{\text {lab }}$. Similar plots have been obtained for the other proton angles. For $\vartheta_{p}^{\text {lab }}=45^{\circ}-125^{\circ}$, the proton efficiency is nearly constant in the central region of each sector, with an average value of $(94 \pm 1) \%$, and drops sharply near the sector boundaries. At forward angles, the average efficiency decreases dropping to about $(50 \pm 1) \%$ at $\vartheta_{p}^{\mathrm{lab}}=15^{\circ}$.

In order to check the reliability of the simulations, the proton detection efficiency has also been obtained using the data (where they are available) from the overdetermined $\gamma d \rightarrow p p \pi^{-}$reaction. Each time a $p$ and $\pi^{-}$pair is found, the 


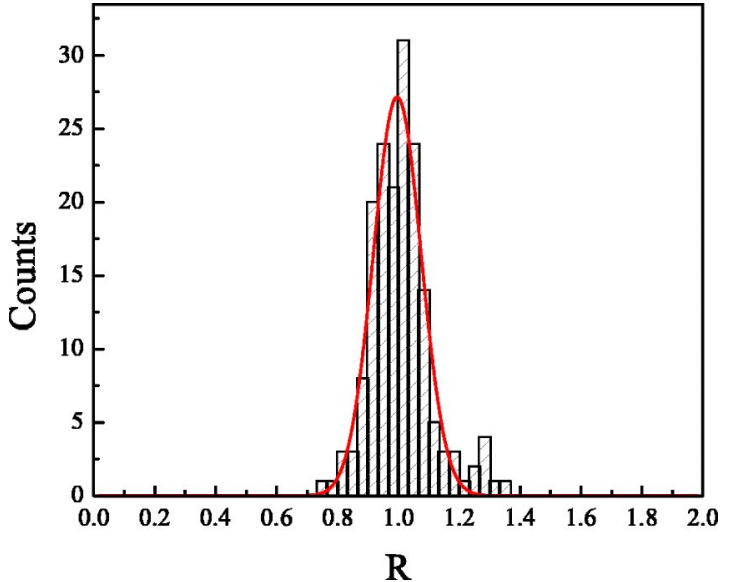

FIG. 3. (Color online) Distribution of the ratio $R=\epsilon_{\mathrm{data}} / \epsilon_{\mathrm{GSIM}}$ between the proton detection efficiency measured from the data using the $\gamma d \rightarrow p \pi^{-} p$ reaction and that obtained from GSIM, for proton momenta in the interval $0.5-1.1 \mathrm{GeV}$ and for the central $20^{\circ}$ in $\varphi$ for all sectors.

missing mass is calculated and the three-momentum is computed for candidates within tight constraints on the proton missing mass. The ratio between the number of exclusive $p \pi^{-} p$ events, $N^{p \pi^{-} p}$, found by the particle identification, and the number of events with $X$ identified as a proton by the missing mass cuts, $N^{p \pi^{-} X(p)}$, minus the number of the background events under the missing mass peak of the $p \pi^{-} X$ distribution, $N_{B}$, gives the experimental detection efficiency:

$$
\epsilon_{\text {data }}=\frac{N^{p \pi^{-} p}}{N^{p \pi^{-} X(p)}-N_{B}} .
$$

The distribution of the values of the ratio $R=\epsilon_{\mathrm{data}} / \epsilon_{\mathrm{GSIM}}$ calculated where both $\epsilon_{\mathrm{data}}$ and $\epsilon_{\mathrm{GSIM}}$ are available with good statistics (proton momenta in the range $0.5-1.1 \mathrm{GeV}$ and central regions of the six CLAS sectors) is shown in Fig. 3.

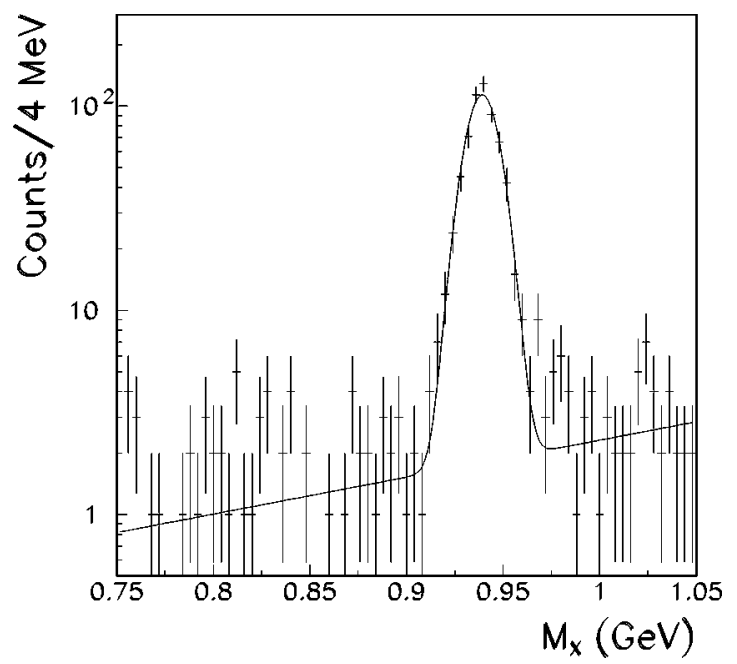

FIG. 4. Typical missing-mass spectrum of the reaction $\gamma d$ $\rightarrow p X$ obtained in Sector 5 for photon energy of $E_{\gamma}=0.95 \mathrm{GeV}$ and proton scattering angle $\vartheta_{p}^{\text {lab }}=25^{\circ}$.

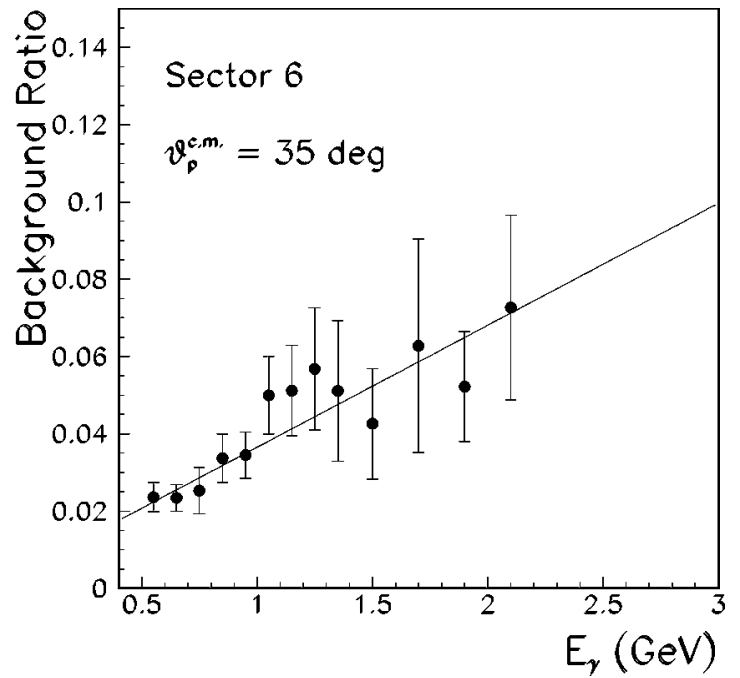

FIG. 5. The behavior of the background contribution $k$ as a function of the photon energy for proton scattering angles $30^{\circ}$ $\leqslant \vartheta_{p}^{\text {c.m. }} \leqslant 40^{\circ}$ and CLAS Sector 6 .

The mean value of the distribution is very close to unity $(0.997 \pm 0.003)$.

We checked also that regions of lower efficiency in CLAS corresponding to dead time-of-flight paddles or drift chambers wires are well reproduced by the simulation. Thus, the comparison over limited kinematics validates the GSIM results.

\section{E. Fiducial cuts and mean efficiencies}

As shown in Fig. 2 the proton detection efficiency is constant in the central azimuthal regions of the six CLAS sectors and decreases steeply near the sector boundaries. Thus, only events in a fiducial region (i.e., azimuthal region of the phase space where the efficiency is uniform) of the detector have

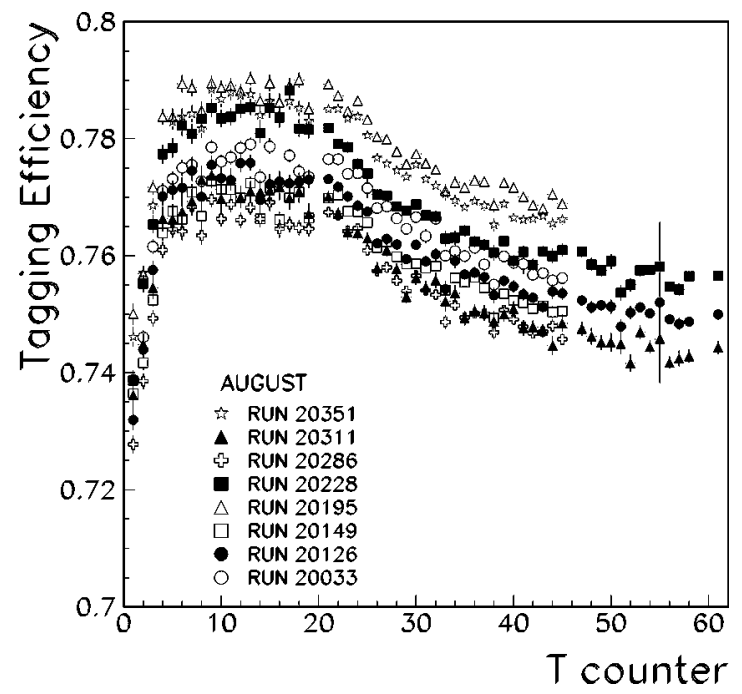

FIG. 6. Tagging efficiency for the 61 tagger-timing counters, measured during the $E_{0}=2.5 \mathrm{GeV}$ normalization runs. In some runs (open points) the low energy counters had been switched off to increase the statistics at high photon energies. 

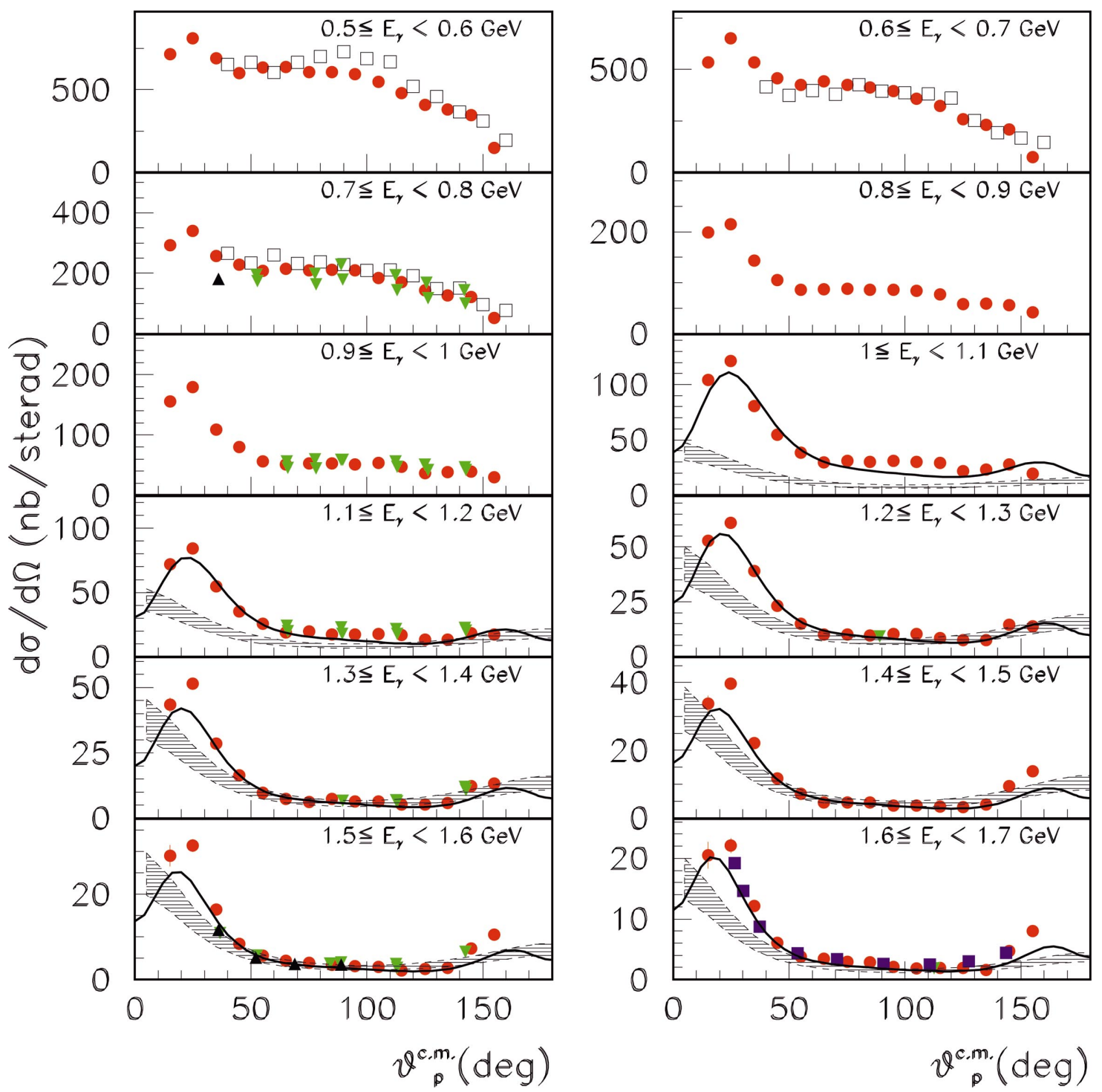

FIG. 7. (Color) Angular distributions of the deuteron photodisintegration cross section measured by the CLAS (full/red circles) in the incident photon energy range $0.50-1.70 \mathrm{GeV}$. Results from Mainz [26] (open squares, average of the measured values in the given photon energy intervals), SLAC [5-7] (full/green down-triangles), JLab Hall A [10] (full/blue squares), and Hall C [8,9] (full/black up-triangles) are also shown. Error bars represent the statistical uncertainties only. The solid line and the hatched area represent the predictions of the QGS [18] and the HRM [27] models, respectively.

been used. For each bin in proton momentum and scattering angle, and for each CLAS sector $S$, a mean efficiency $\epsilon$ is defined as

$$
\epsilon\left(\Delta P_{p}^{\mathrm{lab}}, \Delta \vartheta_{p}^{\mathrm{lab}}, S\right)=\left\langle\epsilon_{\mathrm{GSIM}}\left(\Delta P_{p}^{\mathrm{lab}}, \Delta \vartheta_{p}^{\mathrm{lab}}, S\right)\right\rangle \eta\left(\Delta P_{p}^{\mathrm{lab}}, \Delta \vartheta_{p}^{\mathrm{lab}}, S\right) .
$$

in which $\left\langle\epsilon_{\mathrm{GSIM}}\left(\Delta P_{p}^{\mathrm{lab}}, \Delta \vartheta_{p}^{\mathrm{lab}}, S\right)\right\rangle$ is the average proton detection efficiency over the fiducial $\Delta \varphi$ region, and $\eta\left(\Delta P_{p}^{\mathrm{lab}}, \Delta \vartheta_{p}^{\mathrm{lab}}, S\right)$ is the portion of the CLAS sector inside the fiducial cuts (i.e., the fraction of the $\varphi$ interval considered).

\section{F. Background subtraction}

At all proton angles and photon energies, the missingmass distributions of the $\gamma d \rightarrow p X$ reaction show a neutron mass peak $M_{n}$ riding on a smooth background. As an example, Fig. 4 shows the missing-mass distribution obtained for incident photon energy $E_{\gamma}=0.95 \mathrm{GeV}$ and proton scattering angle $\vartheta_{p}^{\text {lab }}=25^{\circ}$. The logarithmic scale emphasizes the background contribution. These distributions are well reproduced with a Gaussian plus exponential form. Events within $\pm 3 \sigma$ of the neutron peak have been kept for the determination of the cross section (here $\sigma$ is the width of the Gaussian distribution).

The background contribution $N_{B}$ to the number of total events under the peak $N_{\text {peak }}$ has been evaluated by integrating the exponential fit function between the missing-mass cuts. At photon energies higher than $2.0-2.4 \mathrm{GeV}$, depending on the proton angle, the neutron mass peaks are less clearly identifiable due to the low statistics. In these cases, the missing-mass cuts for the selection of exclusive events have 

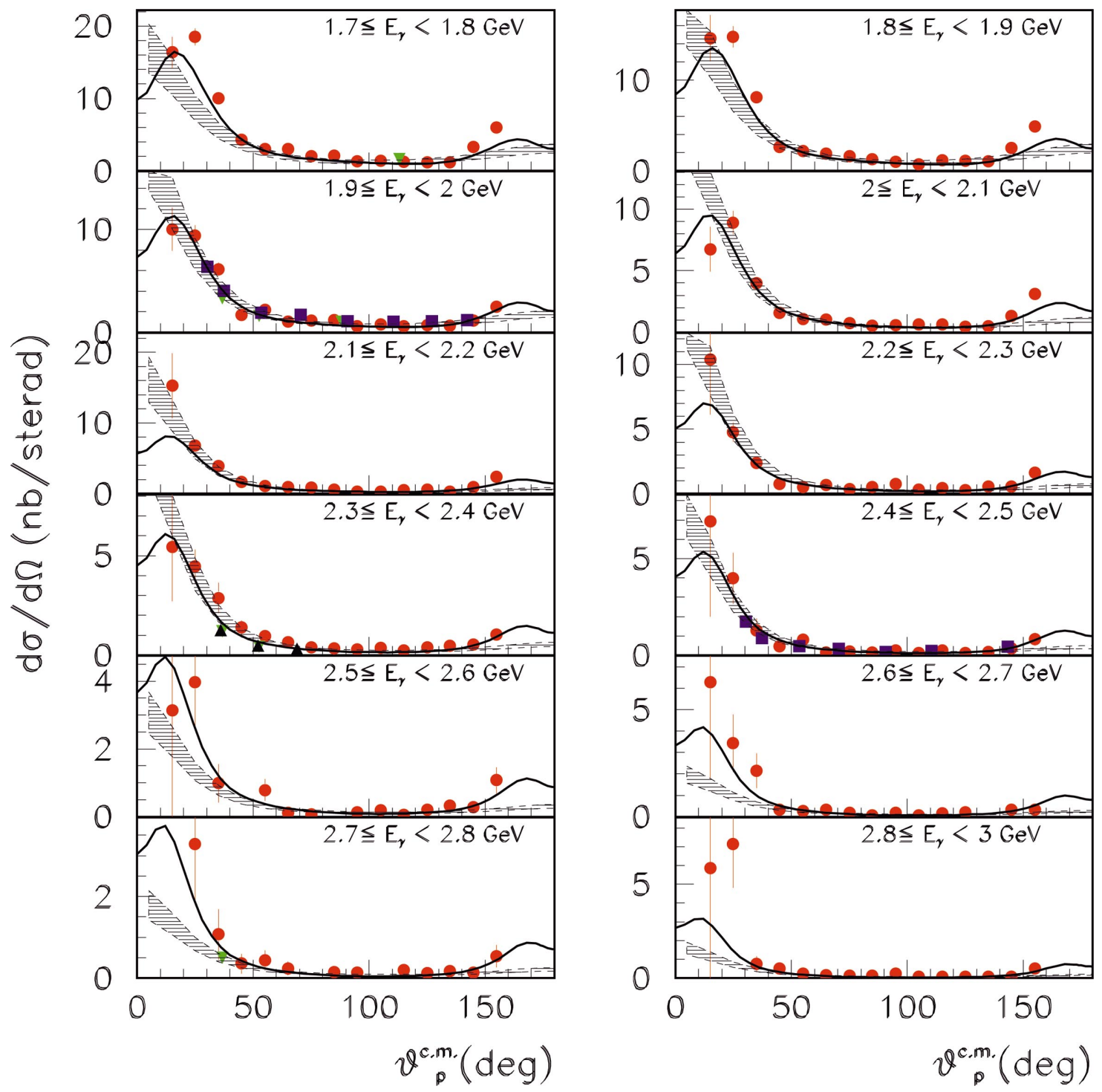

FIG. 8. (Color) Same as Fig. 7 for photon energies 1.7-3.0 GeV.

been obtained using a second-order polynomial fit in $\vartheta_{p}^{\text {c.m. }}$ and $E_{\gamma}$ of the $\left(M_{\text {peak }}+3 \sigma\right)$ and $\left(M_{\text {peak }}-3 \sigma\right)$ values determined at lower photon energies. In each $\vartheta_{p}^{\text {c.m. }}$ bin the background has been evaluated by using a linear extrapolation of the fits in $E_{\gamma}$ to the ratio $k=N_{B} / N_{\text {peak }}$ obtained at lower photon energies. As an example, Fig. 5 shows the values of the ratios $k$ obtained for $30^{\circ} \leqslant \vartheta_{p}^{\text {c.m. }} \leqslant 40^{\circ}$ for CLAS Sector 6 . Similar plots are obtained for other proton angles and CLAS sectors. Here $k$ increases with the photon energy and proton angle $\vartheta_{p}^{\text {c.m. }}$ due to the loss in momentum resolution.

In order to check the extrapolation procedure in the photon energy region above $2.0-2.4 \mathrm{GeV}$, the background contribution has been evaluated from the data using larger bins (then increasing the statistics and making clearly identifiable the peaks) and compared to the result obtained from the extrapolation. The values have been found to be in very good agreement with each other.

\section{G. Photon flux}

The incident flux of photons on the target is given by $N_{\gamma}=N_{e} \epsilon_{T}$, where $N_{e}$ is the number of tagged electrons, and $\epsilon_{T}$ is the tagging efficiency. $N_{e}$ has been measured online during the production runs, while $\epsilon_{T}$ has been measured during the normalization runs at low intensity $\left(\sim 10^{5} \gamma / s\right)$ using a nearly $100 \%$ efficiency lead-glass total absorption counter. We assume that $\epsilon_{T}$ remains unchanged during the production runs. Normalization runs were performed every time the experimental conditions for production runs were changed: a total of $178 \times 10^{6}$ normalization events were collected. Figure 6 shows the tagging efficiency measured in all the normalization runs at $E_{0}=2.5 \mathrm{GeV}$. Similar results were obtained in the normalization runs at $E_{0}=3.1 \mathrm{GeV}$. The tagging efficiency is stable at a level of $\simeq 2 \%$.

\section{H. Systematic uncertainties}

The contributions to the overall systematic uncertainty come from (i) the determination of the number of incident photons, $\approx 1.9 \%$, evaluated by looking at the variation of the number of photons per tagger channel in normalization runs; (ii) the determination of the target length and density, 
TABLE I. Differential cross sections $d \sigma / d \Omega$ in $\mathrm{nb} / \mathrm{sr}$ of the deuteron photodisintegration for photon energies $0.5-3.0 \mathrm{GeV}$ and for center-of-mass proton angles $10^{\circ} \leqslant \vartheta_{p}^{\text {c.m. }}<90^{\circ}$. The first error is statistical and the second is systematic in each case.

\begin{tabular}{|c|c|c|c|c|}
\hline$\left\langle E_{\gamma}\right\rangle(\mathrm{GeV})$ & $10^{\circ} \leqslant \theta_{p}^{\text {c.m. }}<20^{\circ}$ & $20^{\circ} \leqslant \theta_{p}^{\text {c.m. }}<30^{\circ}$ & $30^{\circ} \leqslant \theta_{p}^{\text {c.m. }}<40^{\circ}$ & $40^{\circ} \leqslant \theta_{p}^{\mathrm{c} . \mathrm{m} .}<50$ \\
\hline 0.560 & $714.5 \pm 16.9 \pm 40.2$ & $811.2 \pm 11.6 \pm 48.3$ & $693.2 \pm 8.6 \pm 58.3$ & $601.1 \pm 7.0 \pm 41.3$ \\
\hline 0.641 & $534.3 \pm 11.6 \pm 30.3$ & $651.0 \pm 8.4 \pm 38.8$ & $533.8 \pm 6.2 \pm 44.9$ & $456.6 \pm 5.1 \pm 31.4$ \\
\hline 0.750 & $291.5 \pm 9.5 \pm 16.8$ & $340.6 \pm 6.9 \pm 20.3$ & $256.3 \pm 4.6 \pm 21.6$ & $228.6 \pm 4.0 \pm 15.8$ \\
\hline 0.864 & $198.9 \pm 5.5 \pm 11.7$ & $215.4 \pm 3.8 \pm 12.8$ & $143.3 \pm 2.4 \pm 12.1$ & $105.6 \pm 1.8 \pm 7.3$ \\
\hline 0.950 & $155.2 \pm 4.1 \pm 9.3$ & $179.1 \pm 2.8 \pm 10.7$ & $109.2 \pm 1.7 \pm 9.2$ & $80.01 \pm 1.28 \pm 5.52$ \\
\hline 1.052 & $104.4 \pm 3.9 \pm 6.4$ & $121.1 \pm 2.6 \pm 7.2$ & $80.57 \pm 1.69 \pm 6.78$ & $54.58 \pm 1.20 \pm 3.77$ \\
\hline 1.142 & $72.04 \pm 3.15 \pm 4.47$ & $84.11 \pm 2.12 \pm 5.02$ & $54.95 \pm 1.35 \pm 4.62$ & $35.43 \pm 0.92 \pm 2.45$ \\
\hline 1.254 & $52.80 \pm 2.91 \pm 3.36$ & $61.08 \pm 1.88 \pm 3.65$ & $39.10 \pm 1.20 \pm 3.29$ & $22.99 \pm 0.77 \pm 1.59$ \\
\hline 1.355 & $43.36 \pm 2.73 \pm 2.83$ & $51.28 \pm 1.75 \pm 3.07$ & $28.43 \pm 1.06 \pm 2.39$ & $16.49 \pm 0.66 \pm 1.14$ \\
\hline 1.449 & $33.71 \pm 2.46 \pm 2.25$ & $39.52 \pm 1.54 \pm 2.36$ & $22.06 \pm 0.86 \pm 1.85$ & $11.80 \pm 0.51 \pm 0.82$ \\
\hline 1.548 & $29.01 \pm 2.52 \pm 1.99$ & $31.39 \pm 1.47 \pm 1.88$ & $16.45 \pm 0.82 \pm 1.38$ & $8.36 \pm 0.46 \pm 0.58$ \\
\hline 1.648 & $20.53 \pm 2.18 \pm 1.44$ & $22.11 \pm 1.26 \pm 1.32$ & $12.12 \pm 0.73 \pm 1.02$ & $6.09 \pm 0.40 \pm 0.42$ \\
\hline 1.751 & $16.39 \pm 2.15 \pm 1.18$ & $18.51 \pm 1.21 \pm 1.11$ & $10.03 \pm 0.70 \pm 0.84$ & $4.31 \pm 0.35 \pm 0.30$ \\
\hline 1.845 & $14.62 \pm 2.56 \pm 1.08$ & $14.79 \pm 1.16 \pm 0.89$ & $8.13 \pm 0.67 \pm 0.68$ & $2.64 \pm 0.29 \pm 0.18$ \\
\hline 1.959 & $10.02 \pm 2.08 \pm 0.76$ & $9.43 \pm 0.99 \pm 0.57$ & $6.13 \pm 0.64 \pm 0.51$ & $1.69 \pm 0.27 \pm 0.12$ \\
\hline 2.051 & $6.74 \pm 1.83 \pm 0.53$ & $8.92 \pm 0.96 \pm 0.54$ & $3.97 \pm 0.52 \pm 0.33$ & $1.58 \pm 0.25 \pm 0.11$ \\
\hline 2.147 & $15.29 \pm 4.62 \pm 1.23$ & $6.80 \pm 0.87 \pm 0.41$ & $3.93 \pm 0.56 \pm 0.33$ & $1.70 \pm 0.27 \pm 0.12$ \\
\hline 2.250 & $10.37 \pm 4.24 \pm 0.86$ & $4.75 \pm 0.75 \pm 0.29$ & $2.37 \pm 0.47 \pm 0.20$ & $0.779 \pm 0.187 \pm 0.055$ \\
\hline 2.331 & $5.44 \pm 2.72 \pm 0.46$ & $4.45 \pm 0.87 \pm 0.27$ & $2.88 \pm 0.76 \pm 0.24$ & $1.39 \pm 0.34 \pm 0.10$ \\
\hline 2.458 & $6.94 \pm 4.95 \pm 0.60$ & $3.98 \pm 1.33 \pm 0.24$ & $1.27 \pm 0.63 \pm 0.11$ & $0.46 \pm 0.265 \pm 0.032$ \\
\hline 2.548 & $3.14 \pm 3.15 \pm 0.28$ & $3.96 \pm 1.32 \pm 0.24$ & $0.989 \pm 0.572 \pm 0.083$ & \\
\hline 2.651 & $6.27 \pm 4.45 \pm 0.57$ & $3.43 \pm 1.35 \pm 0.21$ & $2.15 \pm 0.81 \pm 0.18$ & $0.309 \pm 0.219 \pm 0.022$ \\
\hline 2.749 & & $3.28 \pm 1.50 \pm 0.20$ & $1.07 \pm 0.62 \pm 0.09$ & $0.349 \pm 0.248 \pm 0.025$ \\
\hline 2.886 & $5.86 \pm 5.88 \pm 0.57$ & $7.14 \pm 2.34 \pm 0.43$ & $0.748 \pm 0.434 \pm 0.063$ & $0.503 \pm 0.254 \pm 0.036$ \\
\hline$\left\langle E_{\gamma}\right\rangle(\mathrm{GeV})$ & $50^{\circ} \leqslant \theta_{p}^{\text {c.m. }}<60^{\circ}$ & $60^{\circ} \leqslant \theta_{p}^{\text {c.m. }}<70^{\circ}$ & $70^{\circ} \leqslant \theta_{p}^{\text {c.m. }}<80^{\circ}$ & $80^{\circ} \leqslant \theta_{p}^{\text {c.m. }}<90$ \\
\hline 0.560 & $632.3 \pm 6.0 \pm 33.0$ & $637.9 \pm 5.4 \pm 26.8$ & $608.7 \pm 5.3 \pm 26.4$ & $608.8 \pm 5.1 \pm 19.3$ \\
\hline 0.641 & $426.2 \pm 4.0 \pm 22.3$ & $441.8 \pm 3.7 \pm 18.6$ & $426.6 \pm 3.5 \pm 18.5$ & $413.9 \pm 3.4 \pm 13.2$ \\
\hline 0.750 & $207.3 \pm 3.1 \pm 10.9$ & $215.5 \pm 2.7 \pm 9.1$ & $208.9 \pm 2.6 \pm 9.1$ & $211.3 \pm 2.6 \pm 6.8$ \\
\hline 0.864 & $87.07 \pm 1.38 \pm 4.61$ & $87.84 \pm 1.16 \pm 3.72$ & $89.08 \pm 1.23 \pm 3.90$ & $86.93 \pm 1.10 \pm 2.81$ \\
\hline 0.950 & $56.05 \pm 0.90 \pm 2.98$ & $50.54 \pm 0.71 \pm 2.15$ & $52.91 \pm 0.76 \pm 2.32$ & $52.75 \pm 0.69 \pm 1.72$ \\
\hline 1.052 & $38.11 \pm 0.85 \pm 2.04$ & $29.38 \pm 0.62 \pm 1.25$ & $31.05 \pm 0.66 \pm 1.37$ & $30.14 \pm 0.59 \pm 0.99$ \\
\hline 1.142 & $25.56 \pm 0.66 \pm 1.38$ & $18.68 \pm 0.48 \pm 0.8$ & $19.53 \pm 0.50 \pm 0.86$ & $17.41 \pm 0.43 \pm 0.58$ \\
\hline 1.254 & $15.05 \pm 0.53 \pm 0.82$ & $10.29 \pm 0.37 \pm 0.44$ & $10.10 \pm 0.37 \pm 0.45$ & $9.63 \pm 0.33 \pm 0.32$ \\
\hline 1.355 & $9.77 \pm 0.43 \pm 0.54$ & $7.35 \pm 0.33 \pm 0.32$ & $6.29 \pm 0.30 \pm 0.28$ & $7.19 \pm 0.29 \pm 0.24$ \\
\hline 1.449 & $7.12 \pm 0.37 \pm 0.39$ & $4.68 \pm 0.26 \pm 0.2$ & $4.62 \pm 0.23 \pm 0.21$ & $4.63 \pm 0.23 \pm 0.16$ \\
\hline 1.548 & $5.62 \pm 0.35 \pm 0.31$ & $4.35 \pm 0.28 \pm 0.19$ & $3.95 \pm 0.23 \pm 0.18$ & $3.42 \pm 0.21 \pm 0.12$ \\
\hline 1.648 & $3.80 \pm 0.29 \pm 0.21$ & $3.49 \pm 0.26 \pm 0.15$ & $2.90 \pm 0.20 \pm 0.13$ & $2.83 \pm 0.19 \pm 0.10$ \\
\hline 1.751 & $3.07 \pm 0.27 \pm 0.18$ & $3.04 \pm 0.25 \pm 0.13$ & $1.98 \pm 0.17 \pm 0.09$ & $2.10 \pm 0.17 \pm 0.07$ \\
\hline 1.845 & $2.20 \pm 0.25 \pm 0.13$ & $1.92 \pm 0.22 \pm 0.09$ & $1.58 \pm 0.16 \pm 0.07$ & $1.32 \pm 0.14 \pm 0.05$ \\
\hline 1.959 & $2.17 \pm 0.28 \pm 0.13$ & $1.01 \pm 0.19 \pm 0.05$ & $1.15 \pm 0.15 \pm 0.05$ & $1.19 \pm 0.15 \pm 0.04$ \\
\hline 2.051 & $1.08 \pm 0.18 \pm 0.06$ & $1.05 \pm 0.18 \pm 0.05$ & $0.735 \pm 0.117 \pm 0.034$ & $0.551 \pm 0.099 \pm 0.02$ \\
\hline 2.147 & $1.07 \pm 0.19 \pm 0.06$ & $0.95 \pm 0.168 \pm 0.043$ & $0.830 \pm 0.211 \pm 0.038$ & $0.533 \pm 0.099 \pm 0.02$ \\
\hline 2.250 & $0.507 \pm 0.144 \pm 0.031$ & $0.661 \pm 0.146 \pm 0.03$ & $0.361 \pm 0.116 \pm 0.017$ & $0.503 \pm 0.118 \pm 0.019$ \\
\hline 2.331 & $0.970 \pm 0.281 \pm 0.059$ & $0.647 \pm 0.180 \pm 0.029$ & $0.408 \pm 0.152 \pm 0.019$ & $0.36 \pm 0.149 \pm 0.014$ \\
\hline 2.458 & $0.795 \pm 0.326 \pm 0.049$ & $0.125 \pm 0.125 \pm 0.006$ & $0.195 \pm 0.138 \pm 0.009$ & $0.131 \pm 0.093 \pm 0.005$ \\
\hline 2.548 & $0.785 \pm 0.321 \pm 0.049$ & $0.121 \pm 0.121 \pm 0.006$ & $0.072 \pm 0.072 \pm 0.003$ & \\
\hline 2.651 & $0.257 \pm 0.182 \pm 0.016$ & $0.315 \pm 0.183 \pm 0.015$ & $0.183 \pm 0.134 \pm 0.009$ & $0.063 \pm 0.063 \pm 0.002$ \\
\hline
\end{tabular}


TABLE I. (Continued.)

\begin{tabular}{lcccc}
\hline \hline$\left\langle E_{\gamma}\right\rangle(\mathrm{GeV})$ & $10^{\circ} \leqslant \theta_{p}^{\text {c.m. }}<20^{\circ}$ & $20^{\circ} \leqslant \theta_{p}^{\text {c.m. }}<30^{\circ}$ & $30^{\circ} \leqslant \theta_{p}^{\text {c.m. }}<40^{\circ}$ & $40^{\circ} \leqslant \theta_{p}^{\text {c.m. }}<50$ \\
\hline 2.749 & $0.428 \pm 0.248 \pm 0.028$ & $0.237 \pm 0.168 \pm 0.011$ & & $0.148 \pm 0.105 \pm 0.006$ \\
2.886 & $0.224 \pm 0.225 \pm 0.015$ & $0.134 \pm 0.134 \pm 0.006$ & $0.115 \pm 0.115 \pm 0.005$ & $0.110 \pm 0.110 \pm 0.004$ \\
\hline \hline
\end{tabular}

$\approx 0.5 \%$; (iii) the proton detection efficiency, $(2-8) \%$, evaluated as $\left(\epsilon_{\mathrm{GSIM}}-\epsilon_{\mathrm{DATA}}\right) / \epsilon_{\mathrm{GSIM}}$; and (iv) the background subtraction, around $(1-2) \%$ for photon energies below $1 \mathrm{GeV}$ and higher (up to $\sim 6 \%$ ) at forward and backward angles where the detector resolution and efficiency are worse. The latter has been evaluated by repeating the data analysis using both missing-mass cuts reduced and enlarged by $20 \%$, and looking at the variation of the differential cross section. The resulting total systematic error is $\leqslant 10 \%$ in the whole measured range.

\section{RESULTS}

The photodisintegration cross section was calculated using

$$
\frac{d \sigma}{d \Omega}\left(E_{\gamma}, \vartheta_{p}^{\text {c.m. }}\right)=\frac{A}{\rho x N_{A}} \frac{N_{\text {peak }}^{W}\left(E_{\gamma}, \vartheta_{p}^{\text {c.m. }}\right)}{N_{\gamma}\left(E_{\gamma}\right) \Delta \Omega}\left[1-k\left(E_{\gamma}, \vartheta_{p}^{\text {c.m. }}\right)\right],
$$

where $N_{\text {peak }}^{W}$ is the number of $\gamma d \rightarrow p n$ events weighted by the efficiency, $\Delta \Omega$ is the solid angle, $A$ is the target molecular weight, $N_{A}$ is Avogadro's number, $\rho$ is the target density, and $x$ the target effective length.

In Figs. 7 and 8 the angular distributions $d \sigma / d \Omega$ are shown as a function of $\vartheta_{p}^{\text {c.m. }}$ for photon energy bins $100 \mathrm{MeV}$ wide, in the range from 0.5 up to $3.0 \mathrm{GeV}$, and proton scattering angle bins $10^{\circ}$ wide in the range $10^{\circ}$ $\leqslant \vartheta_{p}^{\text {c.m. }} \leqslant 160^{\circ}$. The data are also given in Tables I and II. They are averaged over the six CLAS sectors. The results obtained by the six CLAS sectors separately are consistent with each other within the systematic errors.

This is the first measurement of the nearly complete angular distributions of the $\gamma d \rightarrow p n$ reaction for photon energies between 0.5 and $3.0 \mathrm{GeV}$. It allows one to investigate the behavior of the cross section in the very forward and backward angular regions. The data show a clear forward/ backward angle asymmetry in the whole range of explored photon energies. At high energies the cross sections increase at very forward and backward angles.

Also shown in Figs. 7 and 8 are the previous data and the predictions of the few available models. For $E_{\gamma}$ $=0.5-0.6 \mathrm{GeV}$, the Mainz data [26] are slightly higher than the CLAS data at intermediate scattering angles. Starting from $E_{\gamma}=0.7-0.8 \mathrm{GeV}$ the comparison can be extended also to the SLAC [5-7] and JLab Hall C [8] data. The CLAS results agree well with the data from these experiments. For $E_{\gamma}=1.6-1.7 \mathrm{GeV}, E_{\gamma}=1.9-2.0 \mathrm{GeV}$, and $E_{\gamma}=2.4-2.5 \mathrm{GeV}$, the CLAS results agree with the angular distributions measured by the JLab Hall A collaboration [10] (the latter cover a smaller range in the proton scattering angle: $26^{\circ} \leqslant \vartheta_{p}^{\text {c.m. }}$ $\leqslant 143^{\circ}$ ), and extend to the very forward and backward angular regions where the cross section increases.

For $E_{\gamma} \geqslant 1.0 \mathrm{GeV}$, the predictions of the QGS model $[18,19]$ are shown in Figs. 7 and 8 (solid curve). This model describes the angular distributions very well, and accounts for the persistent forward/backward angle asymmetry seen in the data by invoking the interference of the isovector and isoscalar components of the photon. The interference is constructive at forward angles and destructive in the backward direction. The most forward points support the presence of the local maximum at about $20^{\circ}$ predicted by the model. The backward points do not extend far enough to check for the presence of the second maximum. Also shown in Figs. 7 and 8 are the predictions of the HRM model [27] (hatched band) calculated using the best angular fit for fixed energy $p n$ scattering data. The bands reflect the poor accuracy of the data. The model agrees reasonably well with data in the central angular region over the whole explored energy range, and is lower at forward and backward angles apart from $E_{\gamma}$ $=1.8-2.5 \mathrm{GeV}$. This agreement suggests a further investigation as, in principle, the HRM model is applicable for energies greater than $\sim 2 \mathrm{GeV}$.

The rich amount of CLAS data has made a detailed study of the power law dependence $s^{-n}$ of the differential cross section $d \sigma / d t$ possible, in order to determine the onset threshold for the appearance of the $s^{-11}$ scaling law predicted by perturbative QCD. This study [22] indicates a proton transverse momentum scaling thresholds of $P_{T}$ $=1.0-1.3 \mathrm{GeV} / \mathrm{c}$ for angles between $60^{\circ}$ and $130^{\circ}$, and 0.6-0.9 GeV/c for forward and backward angles, with a nearly symmetric behavior around $90^{\circ}$.

Figure 9 shows the results of $d \sigma / d t$ (full circles) multiplied by the factor $s^{11}$ predicted by CCR and plotted as a function of $E_{\gamma}$ for the four proton scattering angles for which the predictions from all existing models are available. Also shown in the figure are the previous data: Mainz [26] (open squares), SLAC [5-7] (solid or green down triangles), JLab Hall A [10] (solid or blue squares) and Hall C [8,9] (solid or black up triangles). The two points at the same energy value from Ref. [10] shown in the top panel come from two slightly different proton angles $\left(30.3^{\circ}\right.$ and $\left.37.4^{\circ}\right)$. The HRM model [27] (hatched band) agrees reasonably well with data up to about $4 \mathrm{GeV}$, then tends to be higher at forward angles. The RNA calculation is only available at $\vartheta_{p}^{\text {c.m. }}=90^{\circ}$. The estimate for this figure [28] (dashed lines) is normalized to the datum at $E_{\gamma}=3.16 \mathrm{GeV}$. Other estimates at different angles have been provided in other papers by different authors but suffer from an incorrect normalization [29], and therefore are not shown in the figure. The AMEC model [20] (dotted lines) predicts a slightly different energy dependence. The data at forward angles suggest a slower decrease of the cross section with energy than predicted. Moreover, there is a 
TABLE II. Differential cross sections $d \sigma / d \Omega$ in $\mathrm{nb} / \mathrm{sr}$ of the deuteron photodisintegration for photon energies $0.5-3.0 \mathrm{GeV}$ and for center-of-mass proton angles $90^{\circ} \leqslant \vartheta_{p}^{\text {c.m. }}<160^{\circ}$. The first error is statistical and the second is systematic in each case.

\begin{tabular}{|c|c|c|c|c|}
\hline$\left\langle E_{\gamma}\right\rangle(\mathrm{GeV})$ & $90^{\circ} \leqslant \theta_{p}^{\mathrm{c} . \mathrm{m} .}<100^{\circ}$ & $100^{\circ} \leqslant \theta_{p}^{\text {c.m. }}<110^{\circ}$ & $110^{\circ} \leqslant \theta_{p}^{\text {c.m. }}<120^{\circ}$ & $120^{\circ} \leqslant \theta_{p}^{\mathrm{c} . \mathrm{m} .}<130$ \\
\hline 0.560 & $595.8 \pm 5.1 \pm 23.8$ & $545.4 \pm 5.0 \pm 22.3$ & $477.9 \pm 6.9 \pm 25.9$ & $407.8 \pm 7.1 \pm 23.9$ \\
\hline 0.641 & $394.9 \pm 3.6 \pm 15.9$ & $358.6 \pm 3.1 \pm 14.7$ & $322.1 \pm 3.4 \pm 17.5$ & $258.0 \pm 3.5 \pm 15.2$ \\
\hline 0.750 & $209.3 \pm 2.8 \pm 8.6$ & $185.3 \pm 2.4 \pm 7.7$ & $171.2 \pm 2.6 \pm 9.4$ & $145.0 \pm 2.8 \pm 8.6$ \\
\hline 0.864 & $86.76 \pm 1.18 \pm 3.62$ & $85.01 \pm 1.10 \pm 3.57$ & $77.02 \pm 1.16 \pm 4.26$ & $57.99 \pm 1.18 \pm 3.49$ \\
\hline 0.950 & $50.64 \pm 0.72 \pm 2.16$ & $53.61 \pm 0.70 \pm 2.27$ & $47.33 \pm 0.73 \pm 2.64$ & $37.02 \pm 0.68 \pm 2.25$ \\
\hline 1.052 & $31.12 \pm 0.59 \pm 1.36$ & $30.12 \pm 0.59 \pm 1.29$ & $29.15 \pm 0.59 \pm 1.64$ & $21.77 \pm 0.58 \pm 1.35$ \\
\hline 1.142 & $17.30 \pm 0.41 \pm 0.77$ & $18.01 \pm 0.43 \pm 0.78$ & $17.09 \pm 0.42 \pm 0.97$ & $13.27 \pm 0.43 \pm 0.83$ \\
\hline 1.254 & $10.43 \pm 0.33 \pm 0.48$ & $10.55 \pm 0.34 \pm 0.47$ & $8.62 \pm 0.31 \pm 0.50$ & $7.49 \pm 0.33 \pm 0.48$ \\
\hline 1.355 & $6.36 \pm 0.26 \pm 0.30$ & $6.48 \pm 0.27 \pm 0.29$ & $5.23 \pm 0.24 \pm 0.31$ & $5.21 \pm 0.27 \pm 0.34$ \\
\hline 1.449 & $3.69 \pm 0.20 \pm 0.18$ & $3.79 \pm 0.20 \pm 0.17$ & $3.45 \pm 0.19 \pm 0.21$ & $3.17 \pm 0.21 \pm 0.21$ \\
\hline 1.548 & $3.13 \pm 0.19 \pm 0.16$ & $3.02 \pm 0.19 \pm 0.14$ & $2.17 \pm 0.16 \pm 0.13$ & $2.53 \pm 0.20 \pm 0.17$ \\
\hline 1.648 & $2.17 \pm 0.16 \pm 0.11$ & $1.83 \pm 0.15 \pm 0.09$ & $1.94 \pm 0.15 \pm 0.12$ & $1.97 \pm 0.17 \pm 0.14$ \\
\hline 1.751 & $1.36 \pm 0.13 \pm 0.07$ & $1.39 \pm 0.13 \pm 0.07$ & $1.27 \pm 0.13 \pm 0.08$ & $1.18 \pm 0.13 \pm 0.09$ \\
\hline 1.845 & $1.03 \pm 0.12 \pm 0.06$ & $0.694 \pm 0.099 \pm 0.034$ & $1.22 \pm 0.13 \pm 0.08$ & $1.11 \pm 0.14 \pm 0.08$ \\
\hline 1.959 & $0.633 \pm 0.109 \pm 0.037$ & $0.748 \pm 0.109 \pm 0.037$ & $0.599 \pm 0.103 \pm 0.039$ & $0.719 \pm 0.111 \pm 0.055$ \\
\hline 2.051 & $0.600 \pm 0.105 \pm 0.036$ & $0.643 \pm 0.100 \pm 0.032$ & $0.638 \pm 0.111 \pm 0.042$ & $0.456 \pm 0.086 \pm 0.036$ \\
\hline 2.147 & $0.310 \pm 0.109 \pm 0.019$ & $0.310 \pm 0.072 \pm 0.016$ & $0.479 \pm 0.134 \pm 0.032$ & $0.562 \pm 0.102 \pm 0.045$ \\
\hline 2.250 & $0.766 \pm 0.203 \pm 0.049$ & $0.304 \pm 0.076 \pm 0.016$ & $0.420 \pm 0.086 \pm 0.029$ & $0.284 \pm 0.072 \pm 0.024$ \\
\hline 2.331 & $0.297 \pm 0.094 \pm 0.019$ & $0.367 \pm 0.123 \pm 0.019$ & $0.243 \pm 0.115 \pm 0.017$ & $0.403 \pm 0.107 \pm 0.034$ \\
\hline 2.458 & $0.250 \pm 0.125 \pm 0.017$ & $0.116 \pm 0.083 \pm 0.006$ & $0.236 \pm 0.118 \pm 0.017$ & $0.117 \pm 0.083 \pm 0.010$ \\
\hline 2.548 & $0.134 \pm 0.095 \pm 0.009$ & $0.188 \pm 0.110 \pm 0.010$ & $0.058 \pm 0.058 \pm 0.004$ & $0.191 \pm 0.112 \pm 0.017$ \\
\hline 2.651 & $0.181 \pm 0.105 \pm 0.013$ & $0.056 \pm 0.056 \pm 0.003$ & $0.162 \pm 0.094 \pm 0.012$ & $0.214 \pm 0.108 \pm 0.020$ \\
\hline 2.749 & $0.139 \pm 0.098 \pm 0.010$ & & $0.199 \pm 0.115 \pm 0.015$ & $0.123 \pm 0.087 \pm 0.012$ \\
\hline 2.886 & $0.230 \pm 0.164 \pm 0.018$ & $0.066 \pm 0.066 \pm 0.004$ & $0.066 \pm 0.066 \pm 0.005$ & $0.063 \pm 0.063 \pm 0.006$ \\
\hline$\left\langle E_{\gamma}\right\rangle(\mathrm{GeV})$ & $130^{\circ} \leqslant \theta_{p}^{\text {c.m. }}<140^{\circ}$ & $140^{\circ} \leqslant \theta_{p}^{\text {c.m. }}<150^{\circ}$ & $150^{\circ} \leqslant \theta_{p}^{\text {c.m. }}<160$ & \\
\hline 0.560 & $383.2 \pm 5.5 \pm 18.0$ & $347.4 \pm 12.1 \pm 15.4$ & $149.2 \pm 15.6 \pm 10.8$ & \\
\hline 0.641 & $231.3 \pm 3.2 \pm 10.9$ & $207.7 \pm 4.0 \pm 9.2$ & $73.75 \pm 5.31 \pm 5.33$ & \\
\hline 0.750 & $126.4 \pm 2.5 \pm 6.0$ & $120.7 \pm 3.2 \pm 5.4$ & $53.32 \pm 4.45 \pm 3.85$ & \\
\hline 0.864 & $59.20 \pm 1.14 \pm 2.80$ & $56.80 \pm 1.37 \pm 2.58$ & $41.52 \pm 2.42 \pm 3.00$ & \\
\hline 0.950 & $38.31 \pm 0.74 \pm 1.81$ & $39.69 \pm 0.89 \pm 1.81$ & $29.99 \pm 1.44 \pm 2.17$ & \\
\hline 1.052 & $23.56 \pm 0.65 \pm 1.12$ & $27.65 \pm 0.82 \pm 1.27$ & $19.28 \pm 1.13 \pm 1.39$ & \\
\hline 1.142 & $13.28 \pm 0.46 \pm 0.63$ & $18.36 \pm 0.61 \pm 0.85$ & $17.35 \pm 0.96 \pm 1.25$ & \\
\hline 1.254 & $7.44 \pm 0.35 \pm 0.35$ & $14.58 \pm 0.55 \pm 0.68$ & $13.96 \pm 0.84 \pm 1.01$ & \\
\hline 1.355 & $5.90 \pm 0.31 \pm 0.28$ & $12.09 \pm 0.49 \pm 0.57$ & $13.15 \pm 0.80 \pm 0.95$ & \\
\hline 1.449 & $4.08 \pm 0.25 \pm 0.20$ & $9.34 \pm 0.42 \pm 0.45$ & $13.71 \pm 0.79 \pm 0.99$ & \\
\hline 1.548 & $2.81 \pm 0.22 \pm 0.13$ & $7.29 \pm 0.39 \pm 0.35$ & $10.50 \pm 0.70 \pm 0.76$ & \\
\hline 1.648 & $1.59 \pm 0.15 \pm 0.08$ & $4.66 \pm 0.30 \pm 0.23$ & $8.05 \pm 0.59 \pm 0.58$ & \\
\hline 1.751 & $1.20 \pm 0.13 \pm 0.06$ & $3.33 \pm 0.26 \pm 0.16$ & $5.99 \pm 0.51 \pm 0.43$ & \\
\hline 1.845 & $1.07 \pm 0.14 \pm 0.05$ & $2.55 \pm 0.24 \pm 0.13$ & $4.90 \pm 0.48 \pm 0.35$ & \\
\hline 1.959 & $0.692 \pm 0.114 \pm 0.034$ & $1.15 \pm 0.16 \pm 0.06$ & $2.49 \pm 0.35 \pm 0.18$ & \\
\hline 2.051 & $0.507 \pm 0.110 \pm 0.025$ & $1.33 \pm 0.17 \pm 0.07$ & $3.10 \pm 0.38 \pm 0.22$ & \\
\hline 2.147 & $0.320 \pm 0.080 \pm 0.016$ & $0.942 \pm 0.146 \pm 0.048$ & $2.38 \pm 0.34 \pm 0.17$ & \\
\hline 2.250 & $0.555 \pm 0.134 \pm 0.027$ & $0.552 \pm 0.113 \pm 0.028$ & $1.62 \pm 0.28 \pm 0.12$ & \\
\hline 2.331 & $0.481 \pm 0.139 \pm 0.023$ & $0.531 \pm 0.161 \pm 0.027$ & $1.05 \pm 0.29 \pm 0.08$ & \\
\hline 2.458 & $0.166 \pm 0.096 \pm 0.008$ & $0.364 \pm 0.164 \pm 0.019$ & $0.832 \pm 0.343 \pm 0.06$ & \\
\hline 2.548 & $0.327 \pm 0.135 \pm 0.016$ & $0.278 \pm 0.140 \pm 0.015$ & $1.08 \pm 0.39 \pm 0.08$ & \\
\hline 2.651 & & $0.310 \pm 0.140 \pm 0.017$ & $0.325 \pm 0.189 \pm 0.023$ & \\
\hline
\end{tabular}


TABLE II. (Continued.)

\begin{tabular}{lllll}
\hline \hline$\left\langle E_{\gamma}\right\rangle(\mathrm{GeV})$ & $90^{\circ} \leqslant \theta_{p}^{\text {c.m. }}<100^{\circ}$ & $100^{\circ} \leqslant \theta_{p}^{\text {c.m. }}<110^{\circ}$ & $110^{\circ} \leqslant \theta_{p}^{\text {c.m. }}<120^{\circ}$ & $120^{\circ} \leqslant \theta_{p}^{\text {c.m. }}<130$ \\
\hline 2.749 & $0.166 \pm 0.096 \pm 0.008$ & $0.136 \pm 0.097 \pm 0.007$ & $0.535 \pm 0.276 \pm 0.039$ & \\
2.886 & $0.064 \pm 0.047 \pm 0.003$ & $0.072 \pm 0.073 \pm 0.004$ & $0.516 \pm 0.197 \pm 0.037$ & \\
\hline \hline
\end{tabular}

discrepancy for the highest energy at $60^{\circ}-70^{\circ}$. Surprisingly, the model strongly overestimates data at energies lower than 1.6-2.0 GeV. The QGS model describes the data well at all four proton angles. The largest discrepancy is found at $30^{\circ}-40^{\circ}$ above $3 \mathrm{GeV}$ where it suggests a slower decrease of the cross section with energy than observed.

Clearly, further theoretical developments in this nonperturbative regime would be desirable to understand the transition region between the meson exchange picture and the QCD description of high energy nuclear reactions.

\section{SUMMARY}

Differential cross sections for $\gamma d \rightarrow p n$ have been measured for the first time with a nearly complete angular coverage $\left(10^{\circ} \leqslant \vartheta_{p}^{\text {c.m. }} \leqslant 160^{\circ}\right)$ in the photon energy range from 0.5 to $3.0 \mathrm{GeV}$ using the CLAS detector and the tagged photon beam of Hall B at Jefferson Laboratory. The shapes of the angular distributions $d \sigma / d \Omega$ show a persistent forward/ backward angle asymmetry over the whole explored energy range. The cross sections $d \sigma / d t$ fall by $2-3$ orders of mag-

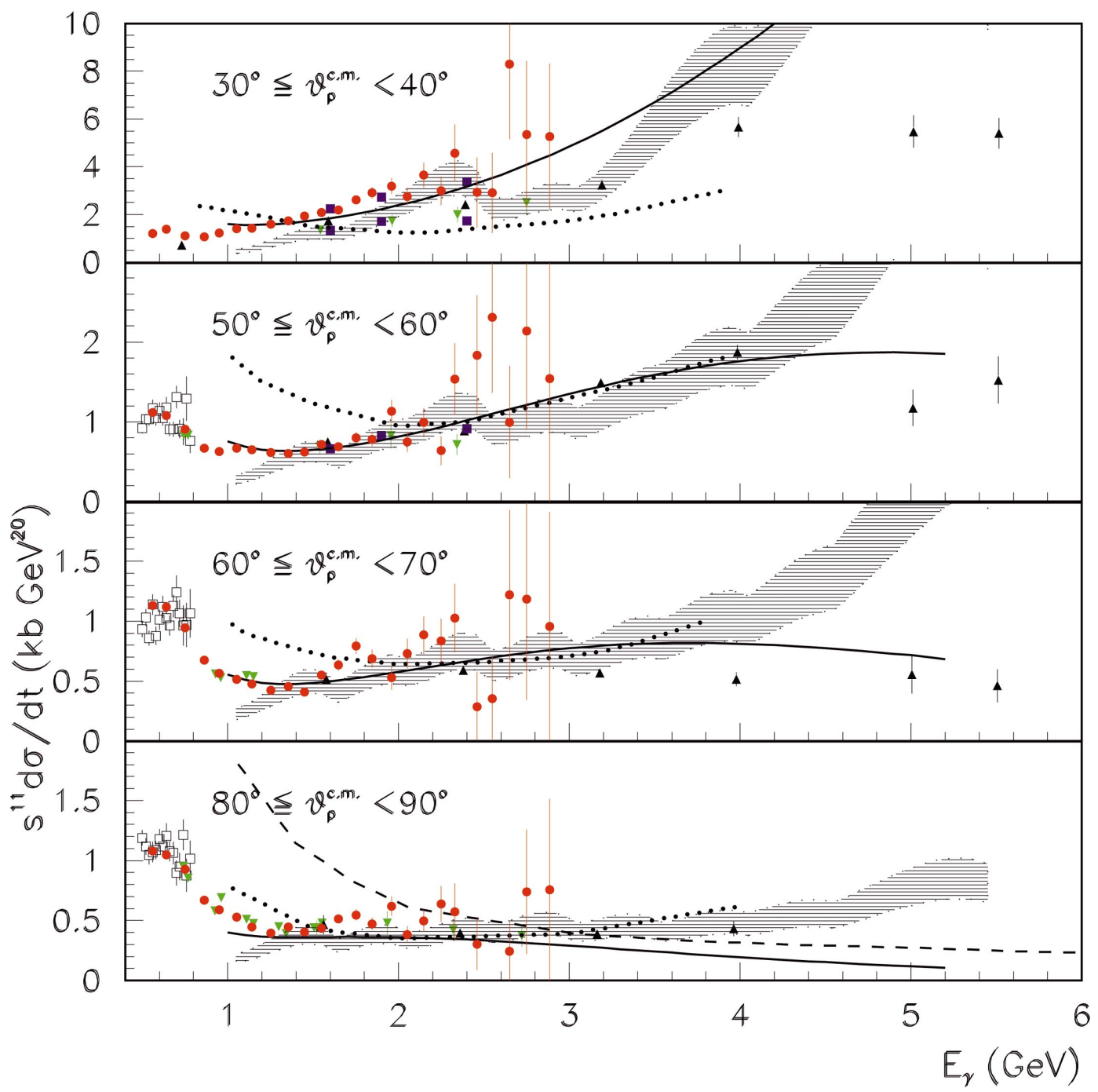

FIG. 9. (Color) Deuteron photodisintegration cross sections $s^{11} d \sigma / d t$ as a function of $E \gamma$ for the given proton scattering angles. Results from CLAS (full/red circles), Mainz [26] (open squares), SLAC [5-7] (full/green down triangles), JLab Hall A [10] (full/blue squares), and Hall C [8,9] (full/black up triangles) are included, as well as predictions of the QGS [18] (solid line), AMEC [20] and RNA [13] models (dotted and dashed lines, respectively), and the HRM [27] model (hatched area). Error bars represent the statistical uncertainties only. 
nitude from 1 to $3 \mathrm{GeV}$ photon energy. The data have been used to determine the scaling threshold at every proton angle between $10^{\circ}$ and $150^{\circ}$. The nonperturbative hard quark rescattering mechanism and quark gluon string models describe the data well. The latter also accounts well for the forward and backward angle asymmetry.

\section{ACKNOWLEDGMENTS}

We would like to acknowledge the outstanding efforts of the staff of the Accelerator and the Physics Divisions at JLab that made this experiment possible. This work was supported in part by the Italian Istituto Nazionale di Fisica Nucleare, the French Centre National de la Recherche Scientifique and the Commissariat à l'Energie Atomique, the U.S. Department of Energy and the National Science Foundation, and the Korea Science and Engineering Foundation. The Southeastern Universities Research Association (SURA) operates the Thomas Jefferson National Accelerator Facility for the United States Department of Energy under Contract No. DE-AC0584ER40150.
[1] R. Gilman and F. Gross, J. Phys. G R37, 28 (2002).

[2] R. J. Holt, Phys. Rev. C 41, 2400 (1990).

[3] V. A. Matveev, R. M. Muradyan, and A. N. Tavkhelidze, Lett. Nuovo Cimento Soc. Ital. Fis. 7, 719 (1973).

[4] S. J. Brodsky and G. Farrar, Phys. Rev. Lett. 31, 1153 (1973).

[5] J. Napolitano et al., Phys. Rev. Lett. 61, 2530 (1988).

[6] S. J. Freedman et al., Phys. Rev. C 48, 1864 (1993).

[7] J. E. Belz et al., Phys. Rev. Lett. 74, 646 (1995).

[8] C. Bochna et al., Phys. Rev. Lett. 81, 4576 (1998).

[9] E. C. Schulte et al., Phys. Rev. Lett. 87, 102302 (2001).

[10] E. C. Schulte et al., Phys. Rev. C 66, 042201 (2002).

[11] K. Wijesooriya et al., Phys. Rev. Lett. 86, 2975 (2001).

[12] F. V. Adamian et al., J. Phys. G 14, 831 (1988).

[13] S. L. Brodsky and J. R. Hiller, Phys. Rev. C 28, 475 (1983).

[14] L. L. Frankfurt, G. A. Miller, M. M. Sargsian, and M. I. Strikman, Phys. Rev. Lett. 84, 3045 (2000).

[15] M. M. Sargsian, Contribution to the Conference "Exclusive Process at High Momentum Transfer," Jefferson Laboratory, 2002, nucl-th/0208027.

[16] B. Julia-Diaz and T. S. H. Lee, Mod. Phys. Lett. A 18, 200 (2003).

[17] L. Kondratyuk, E. De Sanctis, P. Rossi, N. Bianchi, A. B.
Kaidalov, M. J. Krivoruchenko, P. Levi Sandri, V. Muccifora, and A. R. Reolon, Phys. Rev. C 48, 2491 (1993).

[18] V. Y. Grishina, L. Kondratyuk, W. Cassing, A. B. Kaidalov, E. De Sanctis, and P. Rossi, Eur. Phys. J. A 10, 355 (2001).

[19] V. Y. Grishina, L. Kondratyuk, W. Cassing, E. De Sanctis, M. Mirazita, F. Ronchetti, and P. Rossi, Eur. Phys. J. A 19, 117 (2004).

[20] A. E. L. Dieperink and S. I. Nagorny, Phys. Lett. B 456, 9 (1999).

[21] P. Rossi et al., JLab Experiment No. E93-017, 1993.

[22] P. Rossi et al., Phys. Rev. Lett. (submitted).

[23] D. I. Sober et al., Nucl. Instrum. Methods Phys. Res. A 440, 263 (2000).

[24] B. Mecking et al., Nucl. Instrum. Methods Phys. Res. A 503/3, 513 (2003).

[25] M. Mirazita et al., CLAS Analysis Note, 2003.

[26] R. Crawford et al., Nucl. Phys. A603, 303 (1996).

[27] M. M. Sargsian (private communication).

[28] S. J. Brodsky, L. Frankfurt, R. Gilman, J. R. Hiller, G. A. Miller, E. Piasetzky, M. Sargsian, and M. Strikman, Phys. Lett. B 578, 69 (2004).

[29] J. H. Hiller (private communication). 